\title{
Malformaciones urinarias del recién nacido. Estudio ECLAMC 1998-2010
}

\author{
JULIO NAZER H. ${ }^{1}$, LUCÍA CIFUENTES O. ${ }^{2}$, CONSTANZA RAMÍREZ R. ${ }^{3}$ \\ 1. Unidad de Neonatología. Maternidad del Hospital Clínico Universidad de Chile. \\ 2. Programa de Genética Humana, Instituto de Ciencias Biomédicas, Facultad de Medicina. Universidad de Chile. \\ 3. Interna. Facultad de Medicina Universidad de Chile.
}

\begin{abstract}
Urinary malformations among newborns. ECLAMC Registry, 1998-2010

Introduction: Congenital abnormalities of the Urinary Tract are frequent and prevalence has increased since the introduction of routine prenatal sonogram. Objectives: To determine the prevalence rate of congenital urinary malformations at birth at Hospital Clínico de la Universidad de Chile. These data will be compared to other chilean hospitals participating in ECLAMC (Estudio Colaborativo Latino Americano de Malformaciones Congénitas - Latin American Collaborative Study of Congenital Malformations). A longitudinal study will serve to evaluate if significant variation has occurred, and risk factors will be investigated. Patients and Methods: All births occurring between January, 1998 and December, 2010 were included. Prevalence rate of urinary malformations were calculated, and compared to those obtained in previous years. Statistical analysis of proportions was calculated through mean and average comparison was made through Student $t$ test. Results: Urinary anomalies appeared at a rate of 64.5 per 10.000 births. This represents a significant increase from previous studies. Highest risk factor seemed to be "other family members with disease". Most frequent anomaly was Hydroureteronphrosis $(24,2 \%)$ followed by Hypospadias (17\%). The rate of these malformations in ECLAMC participating Chilean hospitales was 23.37/10.000 births. Conclusion: A significant increase in the diagnosis of these anomalies was shown, being the most important risk factor the presence of family members with similar congenital disorders.

(Key words: Urinary congenital malformations, urinary anomalies, ECLAMC, urological congenital disorders, chronic renal failure).

Rev Chil Pediatr 2011; 82 (6): 512-519

\section{RESUMEN}

Introducción: Las anomalías congénitas del Aparato Urinario son frecuentes y ha aumentado su prevalencia al nacimiento con la introducción rutinaria del estudio prenatal por ultrasonografía. Objetivos: Determinar la Tasa de prevalencia al nacimiento de las malformaciones urinarias en el Hospital Clínico de la Universidad de Chile. Compararlas con las del resto de los hospitales chilenos que participan en el ECLAMC (Estudio
\end{abstract}

Trabajo recibido el 28 de junio de 2011, devuelto para corregir el 23 de agosto de 2011, segunda versión el 07 de septiembre de 2011, aceptado para publicación el 04 de octubre de 2011.

Correspondencia a:

Julio Nazer H.

jnazer@redclinicauchile.cl 
Colaborativo Latino Americano de Malformaciones Congénitas). Estudiarlas a lo largo del tiempo para ver si han tenido variaciones significativas. Investigar factores de riesgo que pueden influir en la aparición de ellas. Pacientes y Método: Se estudió todos los nacimientos ocurridos entre Enero de 1998 y Diciembre de 2010 en el Hospital Clínico de la Universidad de Chile. Se calculó las tasas de prevalencia al nacimiento de las malformaciones urinarias y se las comparó con las obtenidas en períodos anteriores. El análisis estadístico de comparación de proporciones se realizó mediante la prueba de $\mathrm{c}^{2} \mathrm{y}$ las comparaciones entre promedios se hicieron mediante prueba t de Student. Resultados: La tasa de Anomalías urinarias fue 64,5 por 10000 nacimientos. Ellas aumentaron significativamente al compararlas con los períodos estudiados anteriormente. El factor de riesgo más influyente fue "otros malformados en la familia". La anomalía más frecuente fue Hidroureteronefrosis $(24,2 \%)$ seguida por Hipospadias (17\%). La tasa de estas malformaciones en los hospitales chilenos participantes del ECLAMC fue 23,37/10 000 nacimientos. Conclusión: Se demuestra un incremento significativo del diagnóstico de estas anomalías, siendo el factor de riesgo más importante el antecedente de otros malformados en la familia.

(Palabras clave: Malformaciones congénitas urinarias, anomalías congénitas, trastornos congénitos urológicos, ECLAMC, insuficiencia renal crónica).

Rev Chil Pediatr 2011; 82 (6): 512-519

\section{Introducción}

Las malformaciones congénitas del Aparato Urinario son frecuentes en el ser humano $\mathrm{y}$ ha aumentado su prevalencia al nacimiento con la introducción rutinaria del estudio prenatal mediante la ultrasonografía, que ha permitido pesquisarlas precozmente. Un estudio europeo demuestra un aumento de $69 \%$ del diagnóstico de estas malformaciones con el uso rutinario de este método al comparar dos decenios ${ }^{1}$. Actualmente, estas anomalías constituyen aproximadamente $20-30 \%$ de todas las malformaciones identificadas en el período prenatal $^{2}$. Las frecuencias diagnosticadas por ultrasonografía en una población no seleccionada se ha estimado entre 0,1 y $0,7 \% 0^{3-6}$. Antes del advenimiento de ella, la mayoría sólo se manifestaban en la niñez o incluso en la vida adulta, al aparecer infecciones urinarias a repetición, hematuria o compromiso de la función renal. Un estudio nacional retrospectivo mostró dentro de una población menor de 1 año con infección urinaria febril, que $20 \%$ presentaba malformaciones urinarias ${ }^{7}$. Se ha estimado que estas anomalías representan un $30-50 \%$ de los casos de insuficiencia renal crónica terminal en la población pediátrica ${ }^{8}$.

En la actualidad debido a los avances en las técnicas de ultrasonografía transvaginal se ha podido visualizar los riñones fetales a partir de la $10^{\text {a }}$ semana de gestación ${ }^{9}$. Por ecografía ab- dominal, sólo se los individualiza a partir de la $12^{\mathrm{a}}$ semana. Es posible evaluar la función renal mediante la visualización de la vejiga, lo que se consigue después de las 11 semanas en 78\% de los exámenes. El 100\% se evidencia desde las 16 semanas?.

Las malformaciones del Sistema Urinario son muy variadas ya que pueden comprometer al riñón, uréteres, vejiga y uretra. Algunas de ellas no tienen tratamiento, pero en la mayoría es posible corregirlas parcial o totalmente. De ahí la importancia de conocerlas y diagnosticarlas precozmente, ojalá prenatalmente o en el período neonatal, especialmente las anomalías obstructivas, ya que permite tratamientos derivativos con el fin de producir la descompresión del sistema urinario y evitar un compromiso mayor del parénquima renal. En los casos de ausencia de riñones o de riñones no funcionantes en que el feto o el RN fatalmente van a fallecer, permite preparar a la madre y su familia ante esa realidad.

Hay otras, consideradas de menor importancia, como el penis cordee o cuerda ventral en que el pene, especialmente el glande, se presenta incurvado por una banda o frenillo rígido. Esta anomalía puede presentarse tanto en niños con hipospadias, como sin hipospadias.

Al revisar los factores de riesgo asociados a las anomalías urinarias, se ha descrito una mayor incidencia en mujeres con historia familiar de malformaciones reno-urinarias ${ }^{10}$. Se las ha 
observado con mayor frecuencia en fetos con anomalías cromosómicas ${ }^{11}$. También se ha comunicado una prevalencia de $3,2 \%$ en pacientes con Síndrome de Down que es mayor que el $0,7 \%$ observado en la población general ${ }^{12}$.

Datos latinoamericanos muestran asociación entre parto prematuro y anomalías como agenesia renal uni o bilateral, enfermedad quística renal e hidronefrosis ${ }^{13}$. Las Hipospadias se han asociado con bajo peso de nacimiento ${ }^{14,15}$. Finalmente, se ha descrito asociación entre malformaciones urinarias con anomalías no renales en un $30 \%$ de los $\operatorname{casos}^{16}$. Esta asociación se ha encontrado en más de 200 síndromes descritos ${ }^{17}$. Kamal Akl encontró en una serie de 30 casos de riñón único o solitario funcionante estudiada, que el $26 \%$ se asociaba con alguna malformación de oído, nariz o garganta y $15 \%$ de malformaciones gastrointestinales.

El Aparato Urinario es parte del Sistema Urogenital, que está constituido, además, por el aparato genital. El primero, también llamado sistema excretor y el segundo sistema reproductor. Ambos sistemas están íntimamente relacionados tanto en su origen embrionario como por sus estructuras anatómicas. Ambos se forman a partir del mesodermo intermedio el que se extiende de manera longitudinal a lo largo de la pared dorsal del cuerpo del embrión ${ }^{18}$.

El sistema urinario está formado por los riñones, que excretan la orina; los uréteres que conducen la orina hacia la vejiga urinaria que la almacena temporalmente y la elimina hacia el exterior a través de la uretra.

\section{Objetivos}

Obtener la Tasa de prevalencia al nacimiento del total de las malformaciones urinarias y de cada una en particular en el Hospital Clinico de la Universidad de Chile. Compararlas con lo observado en el resto de los hospitales chilenos que participan en el ECLAMC (Estudio Colaborativo Latino Americano de Malformaciones Congenitas). Estudiar estas tasas a lo largo del tiempo y evaluar si ha habido variaciones significativas entre ellas. Investigar los factores de riesgo que pueden influir en la aparición de estas anomalias.

\section{Pacientes y Método}

El ECLAMC (Estudio Colaborativo Latino Americano de Malformaciones Congénitas) mantiene en la maternidad del Hospital Clínico de la Universidad de Chile (HCUCH) un Registro de todos los recién nacidos $(\mathrm{RN})$, vivos y mortinatos, de peso de nacimiento de 500 gramos o más, que presentan una o más malformaciones congénitas (MFC). Para los efectos de este trabajo se incluyó a todos los nacimientos consecutivos ocurridos entre el 1 de Enero de 1998 y el 31 de Diciembre de 2010.

Se estudió a todos aquellos niños a los que se diagnosticó, ya sea prenatalmente o posteriormente al nacimiento, alguna malformación renal o uropatías obstructivas. Se calculó las tasas de prevalencia al nacimiento de estas anomalías y se las comparó con las obtenidas en períodos anteriores y publicadas por nosotros $^{6}$. Se compararon los antecedentes de estos niños portadores de malformaciones urinarias con una muestra aleatoria (generada mediante el lanzamiento de dados) de 63 recién nacidos no malformados del mismo período (19982010) de este mismo hospital ${ }^{20}$. El número más pequeño de niños controles que el de casos, obedece a que en nuestra base de datos no están digitalizado los antecedentes de los niños controles, por lo que hay que revisar manualmente cada una de esas fichas.

El análisis estadístico de comparación de proporciones se realizó mediante la prueba de $\chi^{2}$ y las comparaciones entre promedios se hicieron mediante prueba t de Student.

\section{Resultados}

En el período estudiado, 1998-2010, ocurrieron 23689 nacimientos consecutivos en la Maternidad del Hospital Clínico de la Universidad de Chile (HCUCH). De ellos 23495 fueron nacidos vivos (NV) y 194 mortinatos (NM), lo que representa una Mortinatalidad $0,82 \%$. En esta muestra se encontró 2015 recién nacidos $(\mathrm{RN})$ portadores de una o más malformaciones congénitas $(8,5 \%)$. De ellos 1979 eran NV y 65 mortinatos, con lo cual la tasa de prevalencia en NV fue de $8,42 \%$ y 
en NM alcanzó un valor 33,5\%, significativamente mayor ( $\mathrm{p}<0,00001)$. Entre estos RN 153 (64,5 por 10000$)$ presentaban una o más malformaciones del Sistema Urinario. Ciento cuarenta y ocho fueron nacidos vivos y 5 mortinatos. Entre los nacidos vivos $19(12,84 \%)$ fallecieron. Si sumamos los Malformados nacidos vivos que fallecieron y los mortinatos malformados (5), obtenemos una Letalidad de $15,69 \%$ para las malformaciones urinarias.

En la muestra estudiada se encontró 48 malformaciones renales $(26,8 \%) ; 77$ ureterales (43\%); 42 uretrales $(23,5 \%)$; y 10 vesicales $(5,6 \%)$.

La tabla 1 muestra la lista de malformaciones urinarias ordenadas por la frecuencia con que se presentaron en estos niños y la tasa respectiva por 10000 nacimientos. Hidronefrosis e Hidroureteronefrosis son las más frecuentes (24,2\%), le siguen Hipospadias $(17 \%)$, Pielectasia renal $(14,4 \%)$, y Displasia renal multiquística $(13,7 \%)$. Agenesia renal uni o bilateral $(6,5 \%)$, Riñón poliquístico $(3,3 \%)$ y Megavejiga $(3,3 \%)$.

En 23 RN (15\%) la malformación urinaria estaba formando parte de un Síndrome, siendo los más frecuentes Síndrome de Potter y Trisomías 13 y 18 (tabla 2).

En la tabla 3 presentamos la evolución de las tasas de prevalencia al nacimiento de las malformaciones urinarias desde 1971 hasta el 2010, podemos observar como ellas han ido aumentado en forma significativa. Agenesia renal aumentó de 0,37 por 10000 en el período 71-77 a 2,6 en el período 82-97 y a 7,2 en el período 1998-2010, $(\mathrm{p}<0,0001)$. Riñón Poliquístico aumentó de 0.19 en el primer período a $3,3 \%$ en el último ( $p<0,0001)$, Hidronefrosis de 0,37 a $24,2 \%(\mathrm{p}<0,0001)$. La tasa del total de malformados se incrementó de 0,93 por 10000 a 64,5 por $10000(\mathrm{p}<0,0001)$.
Tabla 1. Malformaciones nefro-urinarias en $153 \mathrm{RN}$ del Hospital Clínico de la Universidad de Chile. Período 1998-2010

\begin{tabular}{|lccc|}
\hline Malformación & $\mathbf{n}$ & $\mathbf{\%}$ & $\begin{array}{c}\text { Tasa por } \\
\mathbf{1 0} \mathbf{0 0 0}\end{array}$ \\
\hline Hidronefrosis e Hidroureteronefrosis & 37 & 24,2 & 15,6 \\
\hline Hipospadias & 26 & 17,0 & 11,0 \\
\hline Pielectasia renal & 22 & 14,4 & 9,3 \\
\hline Displasia renal multiquística & 21 & 13,7 & 8,9 \\
\hline Pênis cordee & 12 & 7,8 & 5,1 \\
\hline Agenesia renal & 11 & 7,2 & 4,6 \\
\hline Doble sistema colector & 9 & 5,9 & 3,8 \\
\hline Riñón poliquístico & 5 & 3,3 & 2,1 \\
\hline Megavejiga & 5 & 3,3 & 2,1 \\
\hline Sexo ambíguo & 3 & 2,0 & 1,3 \\
\hline Ectopia renal & 2 & 2,0 & 0,8 \\
\hline Agenesia de vejiga & 2 & 2,0 & 0,8 \\
\hline Cloaca & 2 & 2.0 & 0,8 \\
\hline Megauréter & 2 & 2,0 & 0,8 \\
\hline Epispadias & 2 & 2,0 & 0,8 \\
\hline Hipoplasia renal & 1 & 0,7 & 0,4 \\
\hline Extrofia vesical & 1 & 0,7 & 0,4 \\
\hline Riñón en herradura & 1 & 0,7 & 0,4 \\
\hline Atresia de uretra & 1 & 0,7 & 0,4 \\
\hline Total & 1 & 69,7 \\
\hline
\end{tabular}

Nota: algunos niños tenían más de una malformación.

Tabla 2. Síndromes asociados a malformaciones nefro-urinarias

\begin{tabular}{|lc|}
\hline Diagnóstico & n \\
\hline S de Potter & 11 \\
\hline Trisomía 18 & 3 \\
\hline Trisomía 13 & 3 \\
\hline Sirenomelia & 1 \\
\hline S. de Down & 1 \\
\hline Pseudohermafroditismo masculino & 1 \\
\hline Cri du chat & 1 \\
\hline S. de Turner & 1 \\
\hline S. de Penna- Schokeir & 1 \\
\hline Total & 23 \\
\hline
\end{tabular}


Tabla 3. Evolución de las Tasas de prevalencia al nacimiento de las malformaciones urinarias en el Hospital Clínico de la Universidad de Chile. Períodos 1971-1977, 1982-1997 y 1998-2010.

Tasa por 10000 nacimientos

\begin{tabular}{|c|c|c|c|c|c|c|c|c|}
\hline \multirow{2}{*}{$\begin{array}{l}\text { Período } \\
\text { Malformación }\end{array}$} & \multicolumn{2}{|c|}{ 1971-1977 } & \multicolumn{2}{|c|}{ 1982-1997 } & \multicolumn{2}{|c|}{$1998-2010$} & \multirow[b]{2}{*}{$\chi^{2}$} & \multirow[b]{2}{*}{$\mathbf{p}$} \\
\hline & $\mathbf{n}$ & Tasa & $\mathbf{n}$ & Tasa & $\mathbf{n}$ & Tasa & & \\
\hline Agenesia renal & 2 & 0,37 & 14 & 2,6 & 11 & 4,6 & 14,2 & * \\
\hline Riñón poliquístico & 1 & 0,19 & 35 & 6,5 & 19 & 5,0 & 35,8 & * \\
\hline Hidroureteronefrosis & 2 & 0,37 & 34 & 6,3 & 37 & 24,2 & 84,0 & * \\
\hline Total de malformaciones & 5 & 0,93 & 83 & 15,4 & 165 & 69,7 & 369,4 & * \\
\hline Total de nacimientos & \multicolumn{2}{|c|}{53.931} & \multicolumn{2}{|c|}{54.039} & \multicolumn{2}{|c|}{23.689} & & \\
\hline
\end{tabular}

Tabla 4. Comparación de tres variables demográficas en recién nacidos con malformación urinaria y sus controles

\begin{tabular}{|lccccc|}
\hline Variables & \multicolumn{2}{c}{ MU (n: 153) } & \multicolumn{2}{c|}{ Controles (n: 63) } & Valor p \\
& Promedio & DE & Promedio & DE & \\
\hline Peso al nacer (g) & 3010 & 921,29 & 3364 & 505 & 0,0002 \\
Edad gestacional (s) & 37,25 & 3,22 & 38,4 & 1,3 & 0,0001 \\
\hline Edad materna (a) & 29,84 & 6,22 & 29,7 & 2,4 & 0,5900 \\
\hline
\end{tabular}

DE: desviación estándar. MU: malformación urinaria.

El promedio de peso de nacimiento de los RN malformados fue de 3010 gramos con una dispersión entre 420 gramos y 4590 gramos. Este promedio fue significativamente inferior al de los niños controles $(3364 \mathrm{~g} ; 2,86$, $(\mathrm{p}=0,0002)$. Tabla 4 .

El promedio de edad materna fue de 29,6 años con una dispersión entre 16 y 43 años. Este promedio no difirió del promedio de edad materna de los niños controles (29,7 años; $\mathrm{t}=0,17, \mathrm{p}=0,59)$. Tabla 4 .

El promedio de edad gestacional fue de 37,03 semanas con una dispersión entre 26 y 42 semanas, promedio significativamente inferior al encontrado en los niños controles $(38,4$ semanas; $\mathrm{t}=2,7, \mathrm{p}=0,0001)$. Tabla 4 .

La distribución por sexos demostró un significativo $(\mathrm{p}<0,001)$ predominio masculino: 36 femeninos, 115 masculinos y 3 indiferenciados; Odds ratio para sexo masculino: 3,19 (1,65 a 6,21 con $95 \%$ de confianza).

Entre los factores de riesgo destaca "otros malformados en la familia", ya que 49 $(32,02 \%)$ tenían este antecedente en contraste con sólo $11,1 \%$ de los controles que refieren el antecedente de algún familiar malformado $(\mathrm{p}=0,001)$; Odds ratio: $3,77(1,51$ a 8,79 con 95\% de confianza).

En la tabla 5 se presenta las malformaciones de los familiares afectados. Llama la atención que el 9,8\% (15/153) de los niños con malformaciones urinarias, tenían también un familiar con algún tipo de anomalía urinaria. Igual frecuencia encontramos en malformaciones esqueléticas. Le sigue cardiopatía congéni-

Tabla 5. Malformaciones presentes en los familiares de 153 RN con malformaciones urinarias

\begin{tabular}{|lcc|}
\hline Malformación & n & \% \\
\hline Malformaciones urinarias & 15 & 9,8 \\
\hline Malformaciones esqueléticas & 15 & 9,8 \\
\hline Cardiopatía congénita & 10 & 6,5 \\
\hline Malformaciones del SNC & 4 & 2,6 \\
\hline Retardo mental & 4 & 2,6 \\
\hline Organos de los sentidos & 4 & 2,6 \\
\hline Síndrome de Down & 3 & 2,0 \\
\hline
\end{tabular}


Tabla 6. Tasas de prevalencia al nacimiento de malformaciones urinarias en los hospitales chilenos participantes en el ECLAMC. Período 1998-2008. Tasas por 10000 nacimientos

\begin{tabular}{|c|c|c|c|c|}
\hline Hospitales participantes & Nacimientos & Casos & Tasa & \\
\hline Hospital Clínico Universidad de Chile & 22499 & 127 & 56,45 & $\uparrow$ \\
\hline Regional Valdivia & 11387 & 16 & 14,05 & $\downarrow$ \\
\hline Hospital Clínico Pontificia Universidad Católica de Chile & 1404 & 12 & 85,47 & $\uparrow$ \\
\hline Higueras-Concepción & 13604 & 8 & 5,88 & $\downarrow$ \\
\hline Viña del Mar - Hospital Naval & 8497 & 3 & 3,53 & $\downarrow$ \\
\hline Sanatorio Alemán de Concepción & 4957 & 12 & 24,21 & $\uparrow$ \\
\hline Regional de Rancagua & 52215 & 246 & 47,11 & $\uparrow$ \\
\hline Linares & 24734 & 33 & 13,34 & $\downarrow$ \\
\hline Puerto Montt & 34877 & 42 & 12,04 & $\downarrow$ \\
\hline Sótero del Río-Santiago & 39505 & 157 & 39,74 & $\uparrow$ \\
\hline Salvador de Santiago & 15.864 & 30 & 18,91 & $\downarrow$ \\
\hline San Borja & 34105 & 57 & 16,71 & $\downarrow$ \\
\hline San José de Santiago & 22731 & 41 & 18,03 & $\downarrow$ \\
\hline Talca & 36489 & 49 & 13,42 & $\downarrow$ \\
\hline Cauquenes & 4081 & 3 & 7,35 & $\downarrow$ \\
\hline Curicó & 21401 & 8 & 3,74 & $\downarrow$ \\
\hline Total & 349750 & 844 & 24,13 & \\
\hline
\end{tabular}

$\uparrow \downarrow=$ Tasa significativamente $(p<0,05)$ superior o inferior al promedio nacional.

ta $(6,5 \%)$, tasa muy superior a la encontrada en la población general (1-2\%).

Los antecedentes de enfermedad crónica o aguda de la madre durante el embarazo se presentaron en $50(32,7 \%)$ y en 35 casos $(22,9 \%)$ respectivamente, no más frecuente que lo encontrado en controles $(\mathrm{p}>0,05)$.

Para conocer lo que estaba ocurriendo en el resto de Chile, recurrimos a la base de datos del ECLAMC y con ella construimos las Tablas siguientes.

En la tabla 6 se muestra los Hospitales chilenos participantes en el ECLMC y el total de nacimientos en el período 1998-2008 fue de 352 470. La tasa global encontrada fue de 24,13 por 10000 nacimientos.

Al analizar las Tasas de prevalencia de las anomalías urinarias en los distintos hospitales encontramos una gran heterogeneidad entre ellos, con una dispersión entre 3,53 del Hospital Naval Almirante Neff de Viña del Mar y 85,47 del Hospital Clínico de la Universidad Católica, destacándose además la alta prevalencia de estas malformaciones en los hospitales Clínico de la Universidad de Chile, Regional de Rancagua y Sótero del Río de Santiago.

\section{Discusión}

La prevalencia al nacimiento de malformaciones urinarias obtenida en nuestra muestra $(64,5$ por 10000$)$ está de acuerdo con la encontrada por otros autores en Chile 2 . Otros proponen una prevalencia de 1 a $2 \%{ }^{18}$. En comunicaciones extranjeras Livera ${ }^{4}$ da cifras iguales a la nuestra. Debido al hecho que la mayoría de las malformaciones del aparato urinario son de tipo obstructivo o en forma de quistes, una gran proporción de los diagnósticos de estas patologías pueden ser diagnosticadas prenatalmente y por lo tanto, son dependientes del diagnóstico por ecografía prenatal. Sin ese apoyo es prácticamente imposible hacerlo en el período neonatal, a menos que se palpen masas en las fosas renales que lo hagan sospechar. En efecto, en nuestro material, por ejemplo, la totalidad de las anomalías reno-urinarias, con 
excepción de las Hipospadias y Epispadias, fueron diagnosticadas prenatal mente.

Entre los factores de riesgo estudiados 1lama la atención "Otros malformados en la familia" factor que estuvo presente en $32 \%$ de los casos. Al hacer un análisis de los diagnósticos de las malformaciones en la familia llama la atención que el 9,8\% (15/153) de los niños con malformaciones urinarias, tenían también un familiar con algún tipo de anomalía urinaria. Igual frecuencia encontramos en malformaciones esqueléticas. Le sigue cardiopatía congénita $(6,5 \%)$, tasa muy superior a la encontrada en la población general (1-2\%).

De los otros factores de riesgo, sólo el peso de nacimiento y Edad Gestacional fueron significativamente diferentes entre los casos y los controles, por menor peso y menor Edad Gestacional en los casos $(p<0,05)$. Edad Materna por el contrario no tuvo diferencia significativa $(p>0,05)$. Lo mismo ocurrió con enfermedades agudas y crónicas durante el embarazo $(\mathrm{p}>0,05)$.

La distribución por sexos demostró un significativo predominio masculino $(\mathrm{p}<0,0001)$, ello debido a la presencia de 26 casos de Hipospadias, 12 penis cordee y dos Epispadias (total 40/153; 26,14\%). Al retirar de la muestra las Hipospadias (26), Epispadias (2) y Penis cordee $(12)=40$, esta distribución por sexo, queda con 75 hombres afectados y 36 mujeres, lo cual sigue siendo un predominio significativo de sexo masculino $(\mathrm{p}<0,0001)$.

La tasa de prevalencia al nacimiento de malformaciones urinarias se incrementó en forma muy significativa desde 1971 a 2010 (tabla 3). Esto ocurrió gracias al advenimiento y masificación del diagnóstico prenatal por medio de la Ecografía. En efecto es claro y significativo el incremento de estos diagnósticos a partir de 1982. Después con la introducción de equipos con mejor resolución siguió el aumento hasta la fecha de las tasas de todas las hidroureteronefrosis, riñón poliquístico y multiquístico, no así hipospadia, epispadias.

En la muestra estudiada hidroureteronefrosis fue la anomalía más frecuente, 24,2\% de los casos. Este diagnóstico prenatal es una patología relativamente reciente, ya que antes del advenimiento de la ultrasonografía sólo se realizaba posteriormente, en la niñez o en la vida adulta, por lo general con la aparición de infecciones urinarias a repetición o por signos de insuficiencia renal, por lo que su tratamiento era tardío y por lo tanto, poco eficaz. Pero así como hay casos severos hay otros que son descritos como pielectacias o dilataciones pielocaliciaria muchas de ellas son consideradas transitorias y que necesitan de un seguimiento postnatal antes de ser consideradas como una anormalidad. El tamaño de estas dilataciones (diámetro antero posterior de la pelvis renal) para ser considerado anormal es discutible. Se acepta como tal si mide más de $6 \mathrm{~mm}$ antes de las 20 semanas, más de $8 \mathrm{~mm}$ entre las 20 y las 30 semanas y más de $10 \mathrm{~mm}$ después de las 30 semanas de gestación ${ }^{21}$. Lo habitual en estos casos dudosos es dar de alta al RN con indicaciones de control por urólogo y tratamiento profiláctico con un antibiótico.

Es interesante lo encontrado al analizar las Tasas de prevalencia de las anomalías urinarias en los distintos hospitales chilenos participantes del ECLAMC. Existe una gran heterogeneidad entre ellos, con una dispersión entre 3,53 del Hospital Naval Almirante Neff de Viña del Mar y 85,47 del Hospital Clínico de la Universidad Católica esta última cifra puede estar influenciada por el bajo número de nacimientos registrados en ese centro, destacándose además la alta prevalencia de estas malformaciones en los hospitales Clínico de la Universidad de Chile, Regional de Rancagua y Sótero del Río de Santiago.

\section{Referencias}

1.- Randby H, Meberg A, Yassin H, Tveit L, Watle S, Moe $O$ : Increased detection of malformations of kidneys and the urinary tract. Tidsskr Nor Laegeforen 2009; 129 (19): 1978-80.

2.- Dugoff L: Ultrasound diagnosis of structural abnormalities in the first trimester. Prenat Diagn 2002; 22: 316.

3.- Madarikan BA, Hayward C, Roberts GM, Lari J: Clinical outcome of fetal uropathy. Arch Dis Child 1988; 63: 961.

4.- Livera L Brookfield D, Egginton J, Hawnaur J: Antenatal ultrasonography to detect fetal renal abnormalities: a prospective screening programme. BMJ 1989; 298: 1421. 
5.- Sanghvi KP, Merchant RH, Gondhalekar A, et al: Antenatal diagnosis of congenital renal malformations using ultrasound. J Trop Pediatr 1998; 44: 235.

6.- Nazer J, Fernández P, Silva C: Malformaciones del Aparato Urinário en recién nacidos de la maternidad del Hospital Clínico de la Universidad de Chile. Período 1982-1997. Rev Med Chile 1998; 126: 1472-77.

7.- Bustos P, Arteaga M, Bustamante $M$, et al: Relación entre malformaciones congénitas de la vía urinaria e infecciones del tracto urinario (ITU) bacterémicas en pacientes menores de 1 año hospitalizados en Hospital Clínico San Borja Arriarán (HCSBA) entre 2001 y 2005. Rev Ped Elec 2006; 3, N³.

8.- Seikaly M, Ho P, Emmett L, et al: Chronic renal insufficiency in children: the 2001 Annual Report of the NAPRTCS. Pediatr Nephrol 2003; 18: 796.

9.- Dezerega V, Corral E, Sepúlveda W: Diagnóstico prenatal de malformación urinaria. Rev Chil de Ultrasonografía 2000; 3 (4): 122-33.

10.- Reuss A, Wladimiroff JW, Niermeijer MF: Antenatal diagnosis of renal tract anomalies by ultrasound. Pediatr Nephrol 1987; 1: 546.

11.- Moreno M, Miranda E: Chromosomal Anomalies in Cryptorchidism and Hypospadias. J Urol 2002; 168: 2170.

12.- Kupferman J, Druschel C, Kupchik G: Increased Prevalence of Renal and Urinary Tract Anomalies in Children with Down Syndrome. Pediatrics 2009; 124 (4).

13.- Calderón J, Zarante I: Anomalías congénitas urológi- cas: descripción epidemiológica y factores de riesgo asociados en Colombia 2001-2004. Arch Esp Urol 2006; 59 (1): 7-14.

14.- Weidner I, Moller H, Jensen T: Risk factors for cryptorchidism and hypospadias. J Urol 1999; 161: 1606.

15.- Fredell L, Lichtenstein P, Pedersen $N$ : Hypospadias is related to birth weight in discordant monozygotic twins. J. Urol 1998; 160: 2197.

16.- Wiesel A, Queisser-Luft A, Clementi M, et al: Prenatal detection of congenital renal malformations by fetal ultrasonographic examination: an analysis of 709,030 births in 12 European countries. Eur J Med Genet 2005; 48: 131 .

17.- Limwongse C, Cassidy SB: Syndromes and malformations of the urinary tract. In Pediatric Nephrology, 5th ed, Avner, ED, Harmon, WE, Niaudet, P (Eds), Williams \& Wilkins, Philadelphia 2004. p. 93.

18.- Moore K: Embriología Clínica $4^{a}$ Edición. Ed Interamericana 1988. p 271.

19.- Rosemberg H: Lecciones de Anatomía Patológica. Ed Benedicto Chuaqui. Publicaciones Pontificia Universidad Católica 1996. pag 1-10.

20.- Nazer J, Jarpa C, Cifuentes L: Incidencia y asociaciones de arteria umbilical única em recién nacidos. Maternidad del Hospital Clínico de la Universidad de Chile. Período 1998-2010. Ver Chil Obstet Ginecol 2011; 76 (1): 21-5.

21.- Fefer S, Ellsworth P: Prenatal Hydronefrosis. Pediatr Clin N Am 2006; 53 (3): 429-47. 